\title{
Dynamic Programming for Estimating Acceptance Probability of Credit Card Products
}

\author{
Lai Soon Lee1,2*, Ya Mei Tee', Hsin Vonn Seow ${ }^{3}$ \\ ${ }^{1}$ Department of Mathematics, Faculty of Science, Universiti Putra Malaysia, Serdang, Malaysia \\ ${ }^{2}$ Laboratory of Computational Statistics and Operations Research, Institute for Mathematical Research, \\ Universiti Putra Malaysia, Serdang, Malaysia \\ ${ }^{3}$ Nottingham University Business School, The University of Nottingham Malaysia Campus, \\ Semenyih, Malaysia \\ Email: *1ls@upm.edu.my
}

How to cite this paper: Lee, L.S., Tee, Y.M. and Seow, H.V. (2017) Dynamic Programming for Estimating Acceptance Probability of Credit Card Products. Journal of Computer and Communications, 5, 56-75. https://doi.org/10.4236/jcc.2017.514006

Received: December 1, 2016

Accepted: December 26, 2017

Published: December 29, 2017

Copyright $\odot 2017$ by authors and Scientific Research Publishing Inc. This work is licensed under the Creative Commons Attribution International License (CC BY 4.0).

http://creativecommons.org/licenses/by/4.0/

\begin{abstract}
Banks have many variants of a product which they can offer to their customers. For example, a credit card can have different interest rates. So determining which variants of a product to offer to the new customers and having some indication on acceptance probability will aid with the profit optimisation for the banks. In this paper, the authors look at a model for maximisation of the profit looking at the past information via implementation of the dynamic programming model with elements of Bayesian updating. Numerical results are presented of multiple variants of a credit card product with the model providing the best offer for the maximum profit and acceptance probability. The product chosen is a credit card with different interest rates.
\end{abstract}

\section{Keywords}

Credit Card, Credit Scoring, Dynamic Programming, Profitability

\section{Introduction}

Traditionally, credit card issuers charged a "fixed interest rate" on their credit cards for all their customers. According to [1], since 1991 however, some credit card firms have switched to "variable interest rate" as a result to the credit card lending market becoming more competitive [1]. As reported by [2], profitability of credit card lenders consequently suffered a substantial loss due to this competition.

Hence it is becoming increasing important to be able to secure the acceptance 
of an offer in order to have profit. So, the lenders have to be able to "persuade" the customer to accept their offer. When a good customer is willing to accept an offer, he or she will generate profit to an organisation. One way of doing so is to "customise" the offer to the customer. The lenders could use information about the customer's preferences so as a guide to make a decision on what type of offer the customer may be interested in. This information is already available from initial collection for credit scoring purposes. By looking at which type of product accepted by different customers, the lenders can "learn" about the preferences of their customers. Hence, the decision on what offer to make can be modeled.

There are a number of researchers who have researched acceptance probability for financial products to maximise profitability; for example [3] [4] [5].

In this paper, the authors extended an acceptance model based on the work done by [4]. The lender's decision problem has been modeled as a Markov Decision Process under uncertainty. The objective of this model is the maximisation of profit using a dynamic programming [6] model with Bayesian updating to incorporate the usage of past customer information to optimise acceptance probability. The problem is discussed in the next section. Then the optimal solutions for variants of products are described. Finally, the numerical results are tabled and the conclusions are drawn in the last section.

\section{The Problem}

Banks have many variants of a personal financial product which they can offer to their customers. The attractiveness of the variants to the customer can be ordered in such a way that the likelihood of accepting that variant by the customer is monotonically decreasing while the lender's profitability of the variant is monotonically increasing. For example, a credit card with different interest rates likes $5 \%, 10 \%$ and so on. The decision on which offer to make to the next applicant is based on the given knowledge of the previous offers and whether the offer accepted by previous customers. The objective of modeling the acceptance probability is to maximise the profit to the bank.

In the model here, the authors follow the example of [4] and model the problem as a credit card product with different variant of interest rates. It is assumed that the customers are from homogenous population and the probability of any customer choosing variant $t$ is $p_{t}$ where $t=1,2, \cdots, m$ and $p_{1} \geq p_{2} \geq \cdots \geq p_{m}$. The profit to the lender of $t$ variant chosen by customers is $P_{t}$ where $P_{1} \leq P_{2} \leq \cdots \leq P_{m}$ and $P_{t} \geq 0$. Thus, the bank's maximum profit is determined by $\max \left\{p_{t} P_{t}\right\}$. However, one does not know the probability of $p_{t}$, so we defined $p_{t}$ as with the condition of $p_{1} \geq p_{2} \geq \cdots \geq p_{m}$. Here assume Offer 1 is a credit card with $5 \%$ interest rate annually, Offer 2 is a credit card with $10 \%$ interest rate annually, Offer 3 is a credit card with $15 \%$ interest rate annually and Offer 4 is a credit card with $20 \%$ interest rate annually. Also, assume the number of potential customers has a geometric distribution with parameter $\beta$ with the last customer is $1-\beta$. 
At the beginning, two variants of the credit cards are considered in the model. There are a few assumptions made in this model. First, assume that if a customer rejected variant $t$, meaning that he/she would also reject all worse variants $u$, where $u>t$. Similarly, if a customer accepted variant $t$, he/she would accepted all better variants $v$, where $v<t$. With so $u>t>v$. To illustrate, let $u=20 \%$ interest rate on a credit card, $t=10 \%$ and $v=5 \%$. If one rejects an offer of $10 \%$ interest rate $(t)$, then one is also likely to reject a credit card of $15 \%$ interest rate $(u)$. And if one accepts the offer of $10 \%$ interest rate, one is likely to accept a "better offer" of 5\% $(v)$ interest rate. We ensure this by defining a set of conditional probabilities where $p_{1}$ is the probability of accepting Offer 1 and $q_{1}$ is the Bernoulli random variable.

$q_{1}=p_{1} ;$

$q_{2}=$ Probability (customer would accept Offer 2/customer would accept Offer 1).

Since $q_{1}=p_{1}$,

hence $p_{2}=q_{2} p_{1}=q_{2}\left(q_{1}\right)$.

This condition ensures that $p_{1} \geq p_{2}$.

For three variants of interest rates for the credit card, the conditional probability is as follows:

Since $p_{2}=q_{2} q_{1}$,

hence $p_{3}=q_{3} p_{2}=q_{3}\left(q_{2} q_{1}\right)$ and this ensures that $p_{1} \geq p_{2} \geq p_{3}$.

And so for the four variants of interest rates for the credit card, the conditional probability is as follows:

Since $p_{3}=q_{3} q_{2} q_{1}$,

hence $p_{4}=q_{4} p_{3}=q_{4}\left(q_{3} q_{2} q_{1}\right)$ and this ensures that $p_{1} \geq p_{2} \geq p_{3} \geq p_{4}$.

For many variants of interest rates for the credit card, the conditional probability is defined as:

$$
p_{m}=q_{m} p_{m-1}=\prod_{u=1}^{m} q_{u}
$$

where $p_{m}=$ Probability of accepting offer $m$,

$q_{m}=$ Probability (customer would accept Offer $\mathrm{m} /$ customer would accept Offer $m-1)$.

This condition ensures that $p_{1} \geq p_{2} \geq p_{3} \geq \cdots \geq p_{m}$.

Given that $q_{t}$ are all Bernoulli random variables so in a Bayesian setting, one could describe the bank's knowledge of the information as a Beta distribution. The prior for $q_{t}$ is by $B\left(r_{t}, n_{t}-r_{t}\right)$ whose probability density function is $q_{t}^{r_{t}-1}\left(1-q_{t}\right)^{n_{t}-r_{t}-1}$ and expectation is $\frac{r_{t}}{n_{t}}$ where $r_{t}=$ the number of customers that have accepted the offer $t$ and $n_{t}=$ the number of customers who were extended offer $t$. At any point, the bank's belief about the acceptance probabilities $p_{1} \geq p_{2} \geq p_{3} \geq \cdots \geq p_{m}$ is given by the parameters $\left(r_{1}, n_{1}, r_{2}, n_{2}, \cdots, r_{m}, n_{m}\right)$. Let the expected maximum total future profit to the bank as $V\left(r_{1}, n_{1}, r_{2}, n_{2}, \cdots, r_{m}, n_{m}\right)$ given that the current belief is $\left(r_{1}, n_{1}, r_{2}, n_{2}, \cdots, r_{m}, n_{m}\right)$. 
$r_{1}, n_{1}$ are the parameters of the Beta distribution describing one's belief of $p_{1}$. So if Offer 1 is accepted, the parameters will get updated to $r_{1}+1, n_{1}+1$. If it is rejected, they get updated to $r_{1}, n_{1}+1$. Thus, one could reinterpret these as:

$r_{1}=$ number of customer who already accepted Offer 1 (Offer $5 \%$ in this model); and

$n_{1}=$ number of customer who have been offered Offer 1 (Offer 5\%).

Hence $r_{2}, n_{2}$ are the parameters of the Beta distribution describing one's belief of $p_{2}$. Note the assumption that the offer of Offer 1 will have to be accepted first before Offer 2 can be considered. If Offer 2 is accepted, the parameters get updated to $r_{2}+1, n_{2}+1$. So when it is rejected, they get updated to $r_{2}, n_{2}+1$. Thus,

$r_{2}=$ number of customer who already accepted Offer 2 (Offer $10 \%$ in this model); and

$n_{2}=$ number of customer who have been offered Offer 2 (Offer 10\%).

Note that $r_{3}, n_{3}$ are the parameters of the Beta distribution describing one's belief of $p_{3}$. If Offer 3 is accepted, the parameters get updated to $r_{3}+1, n_{3}+1$. When Offer 3 is rejected, and the customer is assumed to would have accepted Offer 1 but could reject Offer 2; or accepted Offer 1 and Offer 2. Hence they get updated to $r_{3}, n_{3}+1$ and the $r_{1}, n_{1}$ and $r_{2}, n_{2}$ is updated depending on the conditions of Offer 1 and Offer 2 . Thus,

$r_{3}=$ number of customer who already accepted Offer 3 (Offer 15\% in this model); and

$n_{3}=$ number of customer who have been offered Offer 3 (Offer 15\%).

$r_{4}, n_{4}$ are the parameters of the Beta distribution describing one's belief of $p_{4}$. If Offer 4 is accepted, the parameters get updated to $r_{4}+1, n_{4}+1$. If it is rejected, then there are three possibilities:

1) The customer would have accepted Offer 1 but rejected Offer 2 and Offer 3;

2) The customer would have accepted Offer 1 and Offer 2 but rejected Offer 3;

3) The customer would have accepted Offer 1, Offer 2 and Offer 3. Thus,

$r_{4}=$ number of customer who already accepted Offer 4 (Offer $20 \%$ in this model); and

$n_{4}=$ number of customer who have been offered Offer 4 (Offer 20\%).

In the above four cases, $n_{t} \geq r_{t}$ for $t=1,2,3,4$.

By including the information obtained from the past acceptance and rejection of each variants of the product, the model becomes a "learning" model to support making decisions on which product to offer to the next customer.

With such a belief distribution, the expected probability of Offer 1 being accepted is $\frac{r_{1}}{n_{1}}$, Offer 2 is $\frac{r_{1}}{n_{1}} \frac{r_{2}}{n_{2}}$, Offer 3 is $\frac{r_{1}}{n_{1}} \frac{r_{2}}{n_{2}} \frac{r_{3}}{n_{3}}$ and Offer 4 is $\frac{r_{1}}{n_{1}} \frac{r_{2}}{n_{2}} \frac{r_{3}}{n_{3}} \frac{r_{4}}{n_{4}}$. For $k$ offers, this is defined as $\prod_{u=1}^{k} \frac{r_{u}}{n_{u}}$.

Let $V\left(r_{1}, n_{1}, r_{2}, n_{2}\right)=$ expected maximum future profit from the next customer. Consider a two variant case, given that one has to choose which of the two 
variants of the product to offer to the next customer, function $V\left(r_{1}, n_{1}, r_{2}, n_{2}\right)$ has to satisfy the optimal equation (see [7]):

$$
=\max \left\{\begin{array}{l}
V\left(r_{1}, n_{1}, r_{2}, n_{2}\right) \\
\left.+\left(1-\frac{r_{1}}{n_{1}}\right) V\left(r_{1}, n_{1}+1, r_{2}, n_{2}\right)\right\} ; \\
\frac{r_{1}}{n_{1}} \frac{r_{2}}{n_{2}} P_{1}+\beta\left\{\frac{r_{1}}{n_{1}} V\left(r_{1}+1, n_{1}+1, r_{2}, n_{2}\right)\right. \\
+\frac{r_{1}}{n_{1}}\left(1-\frac{r_{2}}{n_{2}} V\left(r_{1}+1, n_{1}+1, r_{2}+1, n_{2}+1\right)\right. \\
+\left(1-\frac{r_{1}}{n_{1}}\right) V\left(r_{1}+1, n_{1}+1, r_{2}, n_{2}+1\right)
\end{array}\right.
$$

For the 3 variants of the credit card product, function $V\left(r_{1}, n_{1}, r_{2}, n_{2}, r_{3}, n_{3}\right)$ satisfies the optimal equation of:

$$
=\max \left\{\begin{array}{l}
\quad\left(1-\frac{r_{1}}{n_{1}}\right) V\left(n_{1}, r_{2}, n_{2}, r_{3}, n_{3}\right) \\
+\left(1-\frac{r_{1}}{n_{1}} P_{1}+\beta\left\{\frac{r_{1}}{n_{1}} V\left(r_{1}+1, n_{1}+1, r_{2}, n_{2}, r_{3}, n_{3}, n_{3}\right)\right\}\right. \\
\frac{r_{1}}{n_{1}} \frac{r_{2}}{n_{2}} P_{2}+\beta\left\{\frac{r_{1}}{n_{1}} \frac{r_{2}}{n_{2}} V\left(r_{1}+1, n_{1}+1, r_{2}+1, n_{2}+1, r_{3}, n_{3}\right)\right. \\
+\frac{r_{1}}{n_{1}}\left(1-\frac{r_{2}}{n_{2}}\right) V\left(r_{1}+1, n_{1}+1, r_{2}, n_{2}+1, r_{3}, n_{3}\right) \\
\frac{r_{1}}{n_{1}} \frac{r_{2}}{n_{2}} \frac{r_{3}}{n_{3}} P_{3}+\beta\left\{\frac{r_{1}}{n_{1}} \frac{r_{2}}{n_{2}} \frac{r_{3}}{n_{3}} V\left(r_{1}+1, n_{1}, r_{3}, n_{3}\right)\right\} \\
+\frac{r_{1}}{n_{1}} \frac{r_{2}}{n_{2}}\left(1-\frac{r_{3}}{n_{3}}\right) V\left(r_{1}+1, n_{1}+1, r_{2}+1, n_{2}+1, r_{3}+1, n_{3}+1\right) \\
+\frac{r_{1}}{n_{1}}\left(1-\frac{r_{2}}{n_{2}}\right) V\left(r_{1}+1, n_{1}+1, r_{2}, n_{2}+1, n_{3}, n_{3}\right) \\
\left.+\left(1-\frac{r_{1}}{n_{1}}\right) V\left(r_{1}, n_{1}+1, r_{2}, n_{2}, r_{3}, n_{3}\right)\right\} .
\end{array}\right.
$$

For the 4 variants of the credit card product, function $V\left(r_{1}, n_{1}, r_{2}, n_{2}, r_{3}, n_{3}, r_{4}, n_{4}\right)$ satisfies the optimal equation of: 


$$
\begin{aligned}
& V\left(r_{1}, n_{1}, r_{2}, n_{2}, r_{3}, n_{3}, r_{4}, n_{4}\right)
\end{aligned}
$$

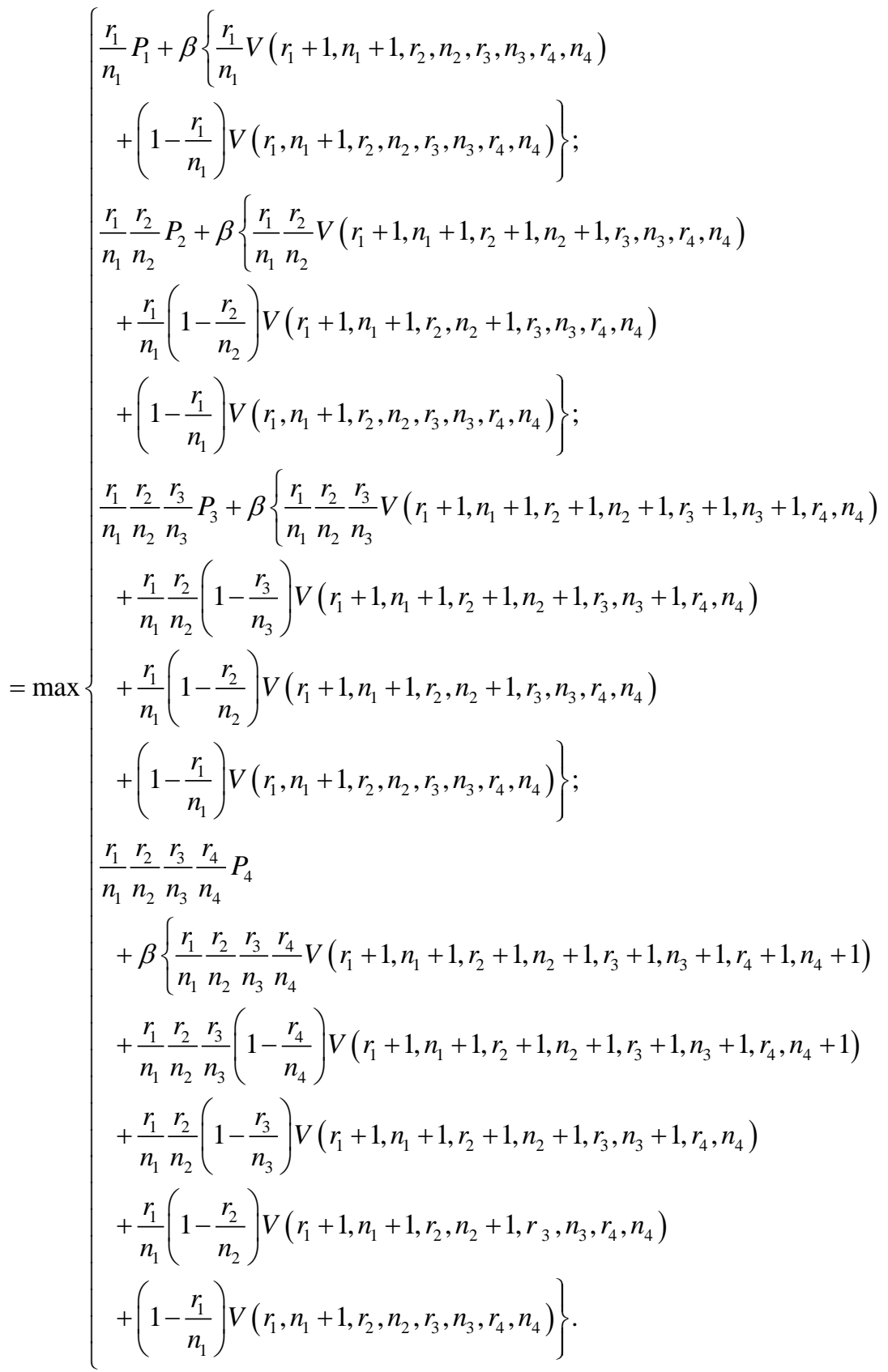

For $m$ variants of products, function $V\left(r_{1}, n_{1}, r_{2}, n_{2}, \cdots, r_{m}, n_{m}\right)$ satisfies the optimal equation:

$$
\begin{aligned}
& V\left(r_{1}, n_{1}, r_{2}, n_{2}, \cdots, r_{m}, n_{m}\right) \\
& =\max _{k: 1 \leq k \leq m}\left\{\prod_{u=1}^{\mathrm{k}}\left(\frac{r_{u}}{n_{u}}\right) P_{k}+\beta \prod_{u=1}^{k}\left(\frac{r_{u}}{n_{u}}\right) V\left(r_{1}+1, n_{1}+1, \cdots, r_{u}+1, n_{u}+1\right)\right. \\
& \left.\quad+\sum_{l=1}^{k}\left(\prod_{u=1}^{k}\left(\frac{r_{u-1}}{n_{u-1}}\right)\left(1-\frac{r_{l}}{n_{l}}\right) V\left(r_{1}+1, n_{1}+1, \cdots, r_{l}, n_{l}+1\right)\right)\right\} .
\end{aligned}
$$

The first term in each offer is the probability that a customer will accept the 
variant offered multiplied by the profit to the bank. The remaining terms depends on the chance $\beta$ that there will be another customer. In the $\beta$ equation, the first term corresponds to the current offer being accepted. The remaining terms correspond to the offer being refused and it looks at the different ways it can happen. For example, the term $V\left(r_{1}+1, n_{1}+1, r_{2}, n_{2}+1\right)$ corresponds to the refusal of the Offer 2. While $V\left(r_{1}, n_{1}+1, r_{2}, n_{2}\right)$ means one believes Offer 1 has been refused thus there is no updating of Offer 2 . The term $V\left(r_{1}+1, n_{1}+1, \cdots, r_{l}, n_{l}+1\right)$ corresponds to the refusal of the 1 -th offer.

\section{Optimal Solution for Many Variants of the Product}

Consider a variation of the problem in (1) where the lender has a cost of $\beta\left(1-\frac{r_{1}}{n_{1}}\right) V\left(r_{1}, n_{1}+1, r_{2}, n_{2}\right)$ if an offer is made to a customer where the state is $\left(r_{1}, n_{1}, r_{2}, n_{2}\right)$ irrespectively of which offer is made. Since the cost is independent of the offer made, it cannot affect the optimal action. Let $\tilde{V}\left(r_{1}, n_{1}, r_{2}, n_{2}\right)$ be the optimal expected profit for the modified problem. Then, we know the optimal policy when solving for $\tilde{V}\left(r_{1}, n_{1}, r_{2}, n_{2}\right)$ is the same as for $V\left(r_{1}, n_{1}, r_{2}, n_{2}\right)$, with

$$
\tilde{V}\left(r_{1}, n_{1}, r_{2}, n_{2}\right)=\frac{r_{1}}{n_{1}} \beta \max \left\{\begin{array}{l}
\frac{B_{1}+\tilde{V}\left(r_{1}+1, n_{1}+1, r_{2}, n_{2}\right) ;}{n_{2}} B_{2}+\frac{r_{2}}{n_{2}} \tilde{V}\left(r_{1}+1, n_{1}+1, r_{2}+1, n_{2}+1\right) \\
+\left(1-\frac{r_{2}}{n_{2}}\right) \tilde{V}\left(r_{1}+1, n_{1}+1, r_{2}, n_{2}+1\right) .
\end{array}\right.
$$

where $B_{i}=\frac{P_{i}}{\beta}, P_{i} \geq 0$, and $i=1,2$.

For the 3 variants case, the optimal expected profit is defined as:

$$
\begin{aligned}
& \tilde{V}\left(r_{1}, n_{1}, r_{2}, n_{2}, r_{3}, n_{3}\right) \\
& =\frac{r_{1}}{n_{1}} \beta \max \left\{\begin{array}{l}
B_{1}+\tilde{V}\left(r_{1}+1, n_{1}+1, r_{2}, n_{2}, r_{3}, n_{3}\right) ; \\
\frac{r_{2}}{n_{2}} B_{2}+\frac{r_{2}}{n_{2}} \tilde{V}\left(r_{1}+1, n_{1}+1, r_{2}+1, n_{2}+1, r_{3}, n_{3}\right) \\
+\left(1-\frac{r_{2}}{n_{2}}\right) \tilde{V}\left(r_{1}+1, n_{1}+1, r_{2}, n_{2}+1, r_{3}, n_{3}\right) ; \\
n_{2} B_{3}+\frac{r_{2}}{n_{2}} \frac{r_{3}}{n_{3}} \tilde{V}\left(r_{1}+1, n_{1}+1, r_{2}+1, n_{2}+1, r_{3}+1, n_{3}+1\right) \\
+\left(1-\frac{r_{3}}{n_{3}}\right) \tilde{V}\left(r_{1}+1, n_{1}+1, r_{2}+1, n_{2}+1, r_{3}, n_{3}+1\right) \\
+\left(1-\frac{r_{2}}{n_{2}}\right) \tilde{V}\left(r_{1}+1, n_{1}+1, r_{2}, n_{2}+1, r_{3}, n_{3}\right) ;
\end{array}\right.
\end{aligned}
$$

where $B_{i}=\frac{P_{i}}{\beta}, \quad i=1,2,3$.

For the 4 variants case, the optimal expected profit is defined as: 


$$
\begin{aligned}
& V\left(r_{1}, n_{1}, r_{2}, n_{2}, r_{3}, n_{3}, r_{4}, n_{4}\right)
\end{aligned}
$$

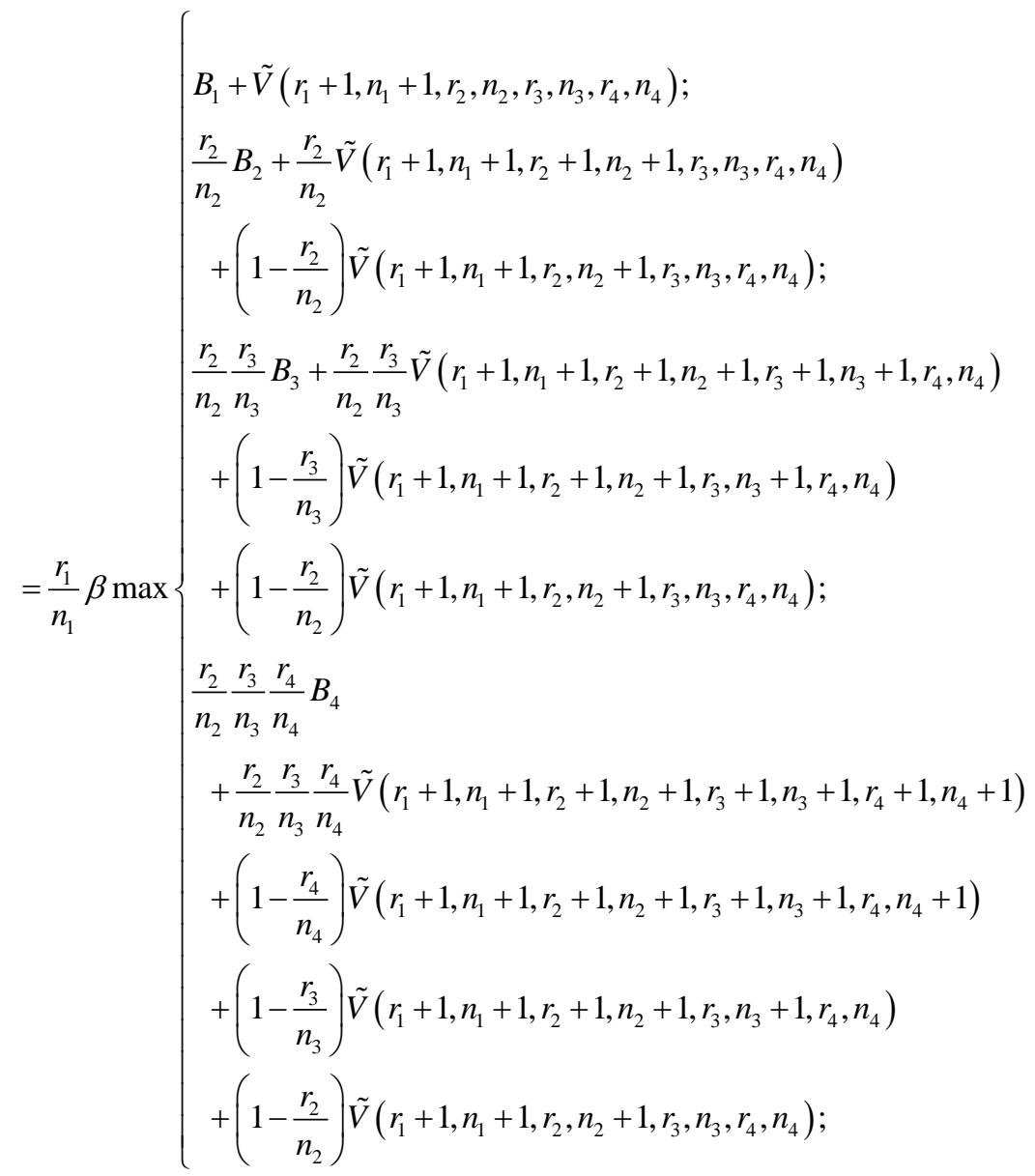

where $B_{i}=\frac{P_{i}}{\beta}, \quad i=1,2,3,4$.

Recall that Equation (4) is the optimal equation for $m$ variants of the product which is the extension of Equations (1)-(3) in the 2, 3 and 4-variants cases respectively. We subtract a cost of $\beta\left(1-\frac{r_{1}}{n_{1}}\right) \tilde{V}\left(r_{1}, n_{1}+1, r_{2}, n_{2}, \cdots, r_{m}, n_{m}\right)$ from all the actions in state of $\left(r_{1}, n_{1}, \cdots, r_{m}, n_{m}\right)$ of Equations (5)-(7). We know that this cannot affect the decisions made but allows us to simplify Equations (5), (6) and (7) to a general equation of:

$$
\begin{aligned}
& \tilde{V}\left(r_{1}, n_{1}, \cdots, r_{m}, n_{m}\right) \\
& =\beta \max _{k: \leq 1 \leq k \leq m}\left\{\prod_{j=1}^{k} \frac{r_{j}}{n_{j}} B_{k}+\prod_{j=1}^{k} \frac{r_{j}}{n_{j}} \tilde{V}\left(r_{1}+1, n_{1}+1, \cdots, r_{k}, n_{k}, r_{k+1}, n_{k+1}\right)\right. \\
& \left.+\sum_{l=2}^{k}\left(\prod_{j=1}^{k}\left(\frac{r_{j-1}}{n_{j-1}}\right)\left(1-\frac{r_{l}}{n_{l}}\right) \tilde{V}\left(r_{1}+1, n_{1}+1, \ldots, r_{l}, n_{l}+1, \cdots, r_{m}, n_{m}\right)\right)\right\} .
\end{aligned}
$$

The proof of the theorem can be referred in Seow and Thomas [6]. If we have 2 variants,

1) $r_{2} \leq r_{1}^{*}\left(r_{2}, n_{2}\right)$, one chooses Offer 1 . 
2) $r_{2}>r_{1}^{*}\left(r_{2}, n_{2}\right)$, one chooses Offer 2 .

If we have 3 variants,

1) $r_{3} \leq r_{2}^{*} \leq r_{1}^{*}\left(r_{2}, n_{2}, r_{3}, n_{3}\right)$, one chooses Offer 1 .

2) $r_{3} \leq r_{2}^{*}\left(r_{3}, n_{3}\right)$, one chooses Offer 2 .

3) $r_{3}>r_{2}^{*}\left(r_{3}, n_{3}\right)$, one chooses Offer 3 .

If we have 4 variants,

1) $r_{4} \leq r_{3}^{*} \leq r_{2}^{*} \leq r_{1}^{*}\left(r_{2}, n_{2}, r_{3}, n_{3}, r_{4}, n_{4}\right)$, one chooses Offer 1 .

2) $r_{4} \leq r_{3}^{*} \leq r_{2}^{*}\left(r_{3}, n_{3}, r_{4}, n_{4}\right)$, one chooses Offer 2 .

3) $r_{4} \leq r_{3}^{*}\left(r_{4}, n_{4}\right)$, one chooses Offer 3 .

4) $r_{4}>r_{3}^{*}\left(r_{4}, n_{4}\right)$, one chooses Offer 4 .

So, if we have $\boldsymbol{m}$ variants, from the theorem as in [4] it is found that:

At any state $\left(r_{1}, n_{1}, \cdots, r_{m}, n_{m}\right)$, there exists functions $r_{i}^{*}\left(r_{i+1}, n_{i+1}, \cdots, r_{m}, n_{m}\right), i=2,3, \cdots, m$ so that:

1) One chooses Offer 1 to all future customers if $r_{2} \leq r_{1}^{*}\left(r_{2}, n_{2}, \cdots, r_{m}, n_{m}\right)$;

2) One chooses Offer 2 to all future customers if $r_{3} \leq r_{2}^{*}\left(r_{3}, n_{3}, \cdots, r_{m}, n_{m}\right)$;

3) One chooses Offer $t$ to all future customers if $r_{t+1} \leq r_{t}^{*}\left(r_{t+1}, n_{t+1}, \cdots, r_{m}, n_{m}\right)$; and

4) One chooses Offer $t+1$ to all future customers if $r_{t+1}>r_{t}^{*}\left(r_{t+1}, n_{t+1}, \cdots, r_{m}, n_{m}\right)$.

We have proved that there is exists at most one

$r_{t}^{*}\left(r_{t+1}, n_{t+1}, \cdots, r_{m}, n_{m}\right), t=1,2,3, \cdots, m$ in the following Lemma 1 .

Lemma 1:

At any state $\left(r_{1}, n_{1}, \cdots, r_{m}, n_{m}\right)$, there is exists at most one $r_{t}^{*}\left(r_{t+1}, n_{t+1}, \cdots, r_{m}, n_{m}\right), t=1,2,3, \cdots, m$ for the choice of $t$ variants of all future customers.

\section{Proof}

To prove the Lemma above, we need to consider two cases.

Case 1 where there is exactly one switch:

1) Let $P(m)$ be the statement that one chooses Offer $t$ to all future customers if $r_{t+1} \leq r_{t}^{*}\left(r_{t+1}, n_{t+1}, \cdots, r_{m}, n_{m}\right)$ and one chooses Offer $t+1$ to all future customers if $r_{t+1}>r_{t}^{*}\left(r_{t+1}, n_{t+1}, \cdots, r_{m}, n_{m}\right)$.

2) Let $P(1)$ which is base offer be the default. Hence for $m=1$, one chooses Offer 1.

3) For $m=2$, assume $P(2)$ is correct, that is:

a) $r_{2} \leq r_{1}^{*}\left(r_{2}, n_{2}\right)$, one chooses Offer 1 .

b) $r_{2}>r_{1}^{*}\left(r_{2}, n_{2}\right)$, one chooses Offer 2 .

Note that there is one $r_{1}^{*}\left(r_{2}, n_{2}\right)$.

For the following statements, $r_{2}^{* *}, r_{3}^{* *}, r_{4}^{* *}, \cdots$ is used to differentiate $r_{2}^{*}, r_{3}^{*}, r_{4}^{*}, \cdots$ since $r_{2}^{* *}, r_{3}^{* *}, r_{4}^{* *}, \cdots$ are not the point to switch the offer.

4) Suppose $P(K)$ is true, for $m=K$, where $P(K)$ is the statement that one chooses Offer $t$ to all future customers if $r_{t+1} \leq r_{t}^{*}\left(r_{t+1}, n_{t+1}, \cdots, r_{K}, n_{K}\right)$; and one chooses Offer $t+1$ to all future customers if $r_{t+1}>r_{t}^{*}\left(r_{t+1}, n_{t+1}, \cdots, r_{K}, n_{K}\right) . P(K)$ also means that:

a) One chooses Offer 1 if $r_{2} \leq r_{1}^{*}\left(r_{2}, n_{2}\right)$.

b) One chooses Offer 1 if $r_{3} \leq r_{2}^{* *} \leq r_{1}^{*}\left(r_{2}, n_{2}, r_{3}, n_{3}\right)$ and one chooses Offer 2 if 
$r_{3} \leq r_{2}^{*}\left(r_{3}, n_{3}\right)$.

c) One chooses Offer 1 if $r_{4} \leq r_{3}^{* *} \leq r_{2}^{* *} \leq r_{1}^{*}\left(r_{2}, n_{2}, r_{3}, n_{3}, r_{4}, n_{4}\right)$; one chooses Offer 2 if $r_{4} \leq r_{3}^{* *} \leq r_{2}^{*}\left(r_{3}, n_{3}, r_{4}, n_{4}\right)$; and one chooses Offer 3 if $r_{4} \leq r_{3}^{*}\left(r_{4}, n_{4}\right)$.

d) One chooses Offer 1 if $r_{t} \leq \cdots \leq r_{4}^{* *} \leq r_{3}^{* *} \leq r_{2}^{* * *} \leq r_{1}^{*}\left(r_{2}, n_{2}, r_{3}, n_{3}, r_{4}, n_{4}, \cdots, r_{K}, n_{K}\right)$; one chooses Offer 2 if $r_{t} \leq \cdots \leq r_{4}^{* *} \leq r_{3}^{* *} \leq r_{2}^{*}\left(r_{3}, n_{3}, r_{4}, n_{4}, \cdots, r_{K}, n_{K}\right)$; one chooses Offer 3 if $r_{t} \leq \cdots \leq r_{4}^{* *} \leq r_{3}^{*}\left(r_{4}, n_{4}, \cdots, r_{K}, n_{K}\right)$; one chooses Offer 4 if $r_{t} \leq \cdots \leq r_{6}^{* *} \leq r_{5}^{* *} \leq r_{4}^{*}\left(r_{5}, n_{5}, r_{6}, n_{6}, \cdots, r_{K}, n_{K}\right)$; and so on, then one chooses Offer $t$ if $r_{t+1} \leq r_{t}^{*}\left(r_{K}, n_{K}\right)$.

e) One chooses Offer $t+1$ if $r_{t+1}>r_{t}^{*}\left(r_{K}, n_{K}\right)$ and there is one $r_{t}^{*}\left(r_{t+1}, n_{t+1}, \cdots, r_{K}, n_{K}\right)$ or switch of offers.

5) It can be shown that $P(K+1)$ is true where $P(K+1)$ is the statement that one chooses Offer $t$ to all future customers if $r_{t+1} \leq r_{t}^{*}\left(r_{t+1}, n_{t+1}, \cdots, r_{K+1}, n_{K+1}\right)$, otherwise one chooses Offer $t+1$ to all future customers if $r_{t+1}>r_{t}^{*}\left(r_{t+1}, n_{t+1}, \cdots, r_{K+1}, n_{K+1}\right)$. $P(K+1)$ also means that:

a) One chooses Offer 1 if $r_{2} \leq r_{1}^{*}\left(r_{2}, n_{2}\right)$.

b) One chooses Offer 1 if $r_{3} \leq r_{2}^{* *} \leq r_{1}^{*}\left(r_{2}, n_{2}, r_{3}, n_{3}\right)$ and one chooses Offer 2 if $r_{3} \leq r_{2}^{*}\left(r_{3}, n_{3}\right)$.

c) One chooses Offer 1 if $r_{4} \leq r_{3}^{* *} \leq r_{2}^{* *} \leq r_{1}^{*}\left(r_{2}, n_{2}, r_{3}, n_{3}, r_{4}, n_{4}\right)$; one chooses Offer 2 if $r_{4} \leq r_{3}^{* *} \leq r_{2}^{*}\left(r_{3}, n_{3}, r_{4}, n_{4}\right)$; and one chooses Offer 3 if $r_{4} \leq r_{3}^{*}\left(r_{4}, n_{4}\right)$.

d) One chooses Offer 1 if

$r_{t+1} \leq \cdots \leq r_{4}^{* *} \leq r_{3}^{* *} \leq r_{2}^{* *} \leq r_{1}^{*}\left(r_{2}, n_{2}, r_{3}, n_{3}, r_{4}, n_{4}, \cdots, r_{K+1}, n_{K+1}\right)$; one chooses Offer 2 if $r_{t+1} \leq \cdots \leq r_{4}^{* *} \leq r_{3}^{* *} \leq r_{2}^{*}\left(r_{3}, n_{3}, r_{4}, n_{4}, \cdots, r_{K+1}, n_{K+1}\right)$; one chooses Offer 3 if $r_{t+1} \leq \cdots \leq r_{4}^{* *} \leq r_{3}^{*}\left(r_{4}, n_{4}, \cdots, r_{K+1}, n_{K+1}\right)$; one chooses Offer 4 if $r_{t+1} \leq \cdots \leq r_{6}^{* *} \leq r_{5}^{* *} \leq r_{4}^{*}\left(r_{5}, n_{5}, r_{6}, n_{6}, \cdots, r_{K+1}, n_{K+1}\right)$; and so on, then one chooses Offer $t$ if $r_{t+1} \leq r_{t}^{*}\left(r_{K+1}, n_{K+1}\right)$.

e) One chooses Offer $t+1$ if $r_{t+1}>r_{t}^{*}\left(r_{K+1}, n_{K+1}\right)$.

Since $P(K)$ is true, that is:

1) One chooses Offer 1 if $r_{2} \leq r_{1}^{*}\left(r_{2}, n_{2}\right)$.

2) One chooses Offer 1 if $r_{3} \leq r_{2}^{* *} \leq r_{1}^{*}\left(r_{2}, n_{2}, r_{3}, n_{3}\right)$ and one chooses Offer 2 if $r_{3} \leq r_{2}^{*}\left(r_{3}, n_{3}\right)$.

3) One chooses Offer 1 if $r_{4} \leq r_{3}^{* *} \leq r_{2}^{* *} \leq r_{1}^{*}\left(r_{2}, n_{2}, r_{3}, n_{3}, r_{4}, n_{4}\right)$; one chooses Offer 2 if $r_{4} \leq r_{3}^{* *} \leq r_{2}^{*}\left(r_{3}, n_{3}, r_{4}, n_{4}\right)$; and one chooses Offer 3 if $r_{4} \leq r_{3}^{*}\left(r_{4}, n_{4}\right)$.

4) One chooses Offer 1 if $r_{t} \leq \cdots \leq r_{4}^{* *} \leq r_{3}^{* *} \leq r_{2}^{* *} \leq r_{1}^{*}\left(r_{2}, n_{2}, r_{3}, n_{3}, r_{4}, n_{4}, \cdots, r_{K}, n_{K}\right)$; one chooses Offer 2 if $r_{t} \leq \cdots \leq r_{4}^{* *} \leq r_{3}^{* *} \leq r_{2}^{*}\left(r_{3}, n_{3}, r_{4}, n_{4}, \cdots, r_{K}, n_{K}\right)$; one chooses Offer 3 if $r_{t} \leq \cdots \leq r_{4}^{* *} \leq r_{3}^{*}\left(r_{4}, n_{4}, \cdots, r_{K}, n_{K}\right)$; one chooses Offer 4 if $r_{t} \leq \cdots \leq r_{6}^{* * *} \leq r_{5}^{* *} \leq r_{4}^{*}\left(r_{5}, n_{5}, r_{6}, n_{6}, \cdots, r_{K}, n_{K}\right)$; and so on, then one chooses Offer $t$ if $r_{t} \leq r_{t-1}^{*}\left(r_{K}, n_{K}\right)$

The introduction of an additional option of choice; the term $r_{t+1} \leq r_{t}^{*}\left(r_{K+1}, n_{K+1}\right)$ in (5, d); can also be expressed as $r_{t+1} \leq r_{t}^{*}\left(r_{K}, n_{K}, r_{K+1}, n_{K+1}\right)$. Since $r_{t+1} \leq r_{t}^{*}\left(r_{K}, n_{K}, r_{K+1}, n_{K+1}\right)$ has an additional option choice of $\left(r_{K+1}, n_{K+1}\right)$, then the next choice is one chooses variant $t$ to all future customers if 
$r_{t+1} \leq r_{t}^{*}\left(r_{K+1}, n_{K+1}\right)$ before it comes to the last condition of choice which is where one chooses variant $t+1$ if $r_{t+1}>r_{t}^{*}\left(r_{K+1}, n_{K+1}\right)$. Hence, $P(K+1)$ is also true, that is:

1) One chooses Offer 1 if $r_{2} \leq r_{1}^{*}\left(r_{2}, n_{2}\right)$.

2) One chooses Offer 1 if $r_{3} \leq r_{2}^{* *} \leq r_{1}^{*}\left(r_{2}, n_{2}, r_{3}, n_{3}\right)$ and one chooses Offer 2 if $r_{3} \leq r_{2}^{*}\left(r_{3}, n_{3}\right)$.

3) One chooses Offer 1 if $r_{4} \leq r_{3}^{* *} \leq r_{2}^{* *} \leq r_{1}^{*}\left(r_{2}, n_{2}, r_{3}, n_{3}, r_{4}, n_{4}\right)$; one chooses Offer 2 if $r_{4} \leq r_{3}^{* *} \leq r_{2}^{*}\left(r_{3}, n_{3}, r_{4}, n_{4}\right)$; and one chooses Offer 3 if $r_{4} \leq r_{3}^{*}\left(r_{4}, n_{4}\right)$.

4) One chooses Offer 1 if

$r_{t+1} \leq \cdots \leq r_{4}^{* *} \leq r_{3}^{* *} \leq r_{2}^{* *} \leq r_{1}^{*}\left(r_{2}, n_{2}, r_{3}, n_{3}, r_{4}, n_{4}, \cdots, r_{K+1}, n_{K+1}\right)$; one chooses Offer 2 if $r_{t+1} \leq \cdots \leq r_{4}^{* *} \leq r_{3}^{* * *} \leq r_{2}^{*}\left(r_{3}, n_{3}, r_{4}, n_{4}, \cdots, r_{K+1}, n_{K+1}\right)$; one chooses Offer 3 if $r_{t+1} \leq \cdots \leq r_{4}^{* *} \leq r_{3}^{*}\left(r_{4}, n_{4}, \cdots, r_{K+1}, n_{K+1}\right)$; one chooses Offer 4 if $r_{t+1} \leq \cdots \leq r_{6}^{* *} \leq r_{5}^{* *} \leq r_{4}^{*}\left(r_{5}, n_{5}, r_{6}, n_{6}, \cdots, r_{K+1}, n_{K+1}\right)$; and so on, then one chooses Offer $t$ if $r_{t+1} \leq r_{t}^{*}\left(r_{K+1}, n_{K+1}\right)$.

5) One chooses Offer $t+1$ if $r_{t+1}>r_{t}^{*}\left(r_{K+1}, n_{K+1}\right)$ and there is one $r_{t}^{*}\left(r_{t+1}, n_{t+1}, \cdots, r_{K+1}, n_{K+1}\right)$.

Case 2 where there is no change in the decision of offer.

If there is no change in the decision of the offer, from above proof of case 1 means that there is only one variant at any state and $r_{t}^{*}\left(r_{t+1}, n_{t+1}, \cdots, r_{m}, n_{m}\right), t=1,2,3, \cdots, \quad m$ does not exist trivially.

\section{Empirical Results and Analysis}

In this section, the data needed to get information for learning the switch of offers has been generated using the dynamic programming model. This is based on expected profit generated (in $£$ ). Some results generated by the model are shown in the following tables. We first defined $\beta=0.5$ for 2 and 3 variants in the model. Then defined $\beta=0.999$ for 4 variants. We have subtracted the "fee" from the model, hence the values shown are not the full profits. Please note the choice of $\beta=0.5$ and 0.999 was based on the purpose to illustrate the profit generated at $50 \%$ discounting factor and almost $100 \%$ discounting factor.

\subsection{Two Variants Case}

If there are 2 variant of products (5\% and $10 \%$ interest rates), then variant $5 \%$ will be chosen if $\frac{r_{1}}{n_{1}} P_{1}>\frac{r_{1}}{n_{1}} \frac{r_{2}}{n_{2}} P_{2}$ and otherwise, variant $10 \%$ will be chosen if $\frac{r_{1}}{n_{1}} \frac{r_{2}}{n_{2}} P_{2}>\frac{r_{1}}{n_{1}} P_{1}$.

Table 1 and Table 2 present some of the results generated by the model. The bold in the row is the point when the switch of offers occurs. We choose $r_{1}=3$ and $n_{1}=10$ to represent a case where one's belief of the acceptance of variant $5 \%$ is $p_{1}=\frac{3}{10}$. 
Table 1. Part of results generated by the acceptance model when $P_{1}=10.000, P_{2}=$ 25.000, $\beta=0.5, m=45, p=10$.

\begin{tabular}{cccccc}
\hline $\boldsymbol{r}_{1}$ & $\boldsymbol{n}_{1}$ & $\boldsymbol{r}_{2}$ & $\boldsymbol{n}_{2}$ & Profit $(\mathfrak{E})$ & Offer \\
\hline 3 & 10 & $0-2$ & 6 & 3.6943 & $5 \%$ \\
3 & 10 & 3 & 6 & 4.6206 & $10 \%$ \\
3 & 10 & 4 & 6 & 6.1572 & $10 \%$ \\
3 & 10 & 5 & 6 & 7.6965 & $10 \%$ \\
3 & 10 & 6 & 6 & 9.2358 & $10 \%$ \\
\hline
\end{tabular}

Table 2. Part of results generated by the acceptance model when $P_{1}=10.000, P_{2}=$ $25.000, \beta=0.5, m=45, p=10$.

\begin{tabular}{|c|c|c|c|c|c|}
\hline$r_{1}$ & $n_{1}$ & $r_{2}$ & $n_{2}$ & Profit $(\mathfrak{E})$ & Offer \\
\hline 3 & 10 & $0-8$ & 22 & 3.6943 & $5 \%$ \\
\hline 3 & 10 & 9 & 22 & 3.7872 & $10 \%$ \\
\hline 3 & 10 & 10 & 22 & 4.1981 & $10 \%$ \\
\hline 3 & 10 & 11 & 22 & 4.6179 & $10 \%$ \\
\hline 3 & 10 & 12 & 22 & 5.0377 & $10 \%$ \\
\hline 3 & 10 & 13 & 22 & 5.4575 & $10 \%$ \\
\hline 3 & 10 & 14 & 22 & 5.8773 & $10 \%$ \\
\hline 3 & 10 & 15 & 22 & 6.2971 & $10 \%$ \\
\hline 3 & 10 & 16 & 22 & 6.7169 & $10 \%$ \\
\hline 3 & 10 & 17 & 22 & 7.1367 & $10 \%$ \\
\hline 3 & 10 & 18 & 22 & 7.5566 & $10 \%$ \\
\hline 3 & 10 & 19 & 22 & 7.9764 & $10 \%$ \\
\hline 3 & 10 & 20 & 22 & 8.3962 & $10 \%$ \\
\hline 3 & 10 & 21 & 22 & 8.8160 & $10 \%$ \\
\hline 3 & 10 & 22 & 22 & 9.2358 & $10 \%$ \\
\hline
\end{tabular}

Table 3 and Table 4 present a case where one's belief of the acceptance of variant $5 \%$ with the ratio of $p_{1}=\frac{1}{2}$ and some of the belief points at which the offer decision changes.

Table 5 and Table 6 present a case where one's belief of the acceptance of variant $5 \%$ with the ratio of $p_{1}=\frac{5}{6}$ and some of the belief points at which the offer decision changes.

Table 7 and Table 8 show an example where there is no any point of the switch of offers occurs. That is $r_{t}^{*}\left(r_{t+1}, n_{t+1}, \cdots, r_{m}, n_{m}\right)$ does not exists in any state. We choose $r_{2}=1$ and $n_{2}=5$ to represent a case where one's belief of the acceptance of variant $10 \%$ is $p_{2}=\frac{1}{5}$. 
Table 3. Changing of offers when $r_{1}=1, n_{1}=2, P_{1}=10.000, P_{2}=25.000, \beta=0.5, m=45$, $p=10$.

\begin{tabular}{ccccccccccc}
\hline $\boldsymbol{r}_{2}$ & 0 & 1 & 2 & 3 & 4 & 5 & 6 & 7 & 8 & 9 \\
\hline $\boldsymbol{n}_{2}$ & $1-2$ & $3-5$ & $6-7$ & $8-10$ & $11-12$ & $13-15$ & $16-17$ & $18-20$ & $21-22$ & $23-24$ \\
\hline
\end{tabular}

Table 4. Changing of offers when $r_{1}=8, n_{1}=16, P_{1}=10.000, P_{2}=25.000, \beta=0.5, m=45$, $p=10$.

\begin{tabular}{ccccccccccc}
\hline $\boldsymbol{r}_{2}$ & 0 & 1 & 2 & 3 & 4 & 5 & 6 & 7 & 8 & 9 \\
\hline $\boldsymbol{n}_{2}$ & $1-2$ & $3-5$ & $6-7$ & $8-10$ & $11-12$ & $13-15$ & $16-17$ & $18-20$ & $21-22$ & $23-24$ \\
\hline
\end{tabular}

Table 5. Changing of offers when $r_{1}=5, n_{1}=6, P_{1}=10.000, P_{2}=25.000, \beta=0.5, m=45$, $p=10$.

\begin{tabular}{ccccccccccc}
\hline $\boldsymbol{r}_{2}$ & 0 & 1 & 2 & 3 & 4 & 5 & 6 & 7 & 8 & 9 \\
\hline $\boldsymbol{n}_{2}$ & $1-2$ & $3-5$ & $6-7$ & $8-10$ & $11-12$ & $13-15$ & $16-17$ & $18-20$ & $21-22$ & $23-24$ \\
\hline
\end{tabular}

Table 6. Changing of offers when $r_{1}=15, n_{1}=18, P_{1}=10.000, P_{2}=25.000, \beta=0.5, m=$ $45, p=10$.

\begin{tabular}{ccccccccccc}
\hline $\boldsymbol{r}_{2}$ & 0 & 1 & 2 & 3 & 4 & 5 & 6 & 7 & 8 & 9 \\
\hline $\boldsymbol{n}_{2}$ & $1-2$ & $3-5$ & $6-7$ & $8-10$ & $11-12$ & $13-15$ & $16-17$ & $18-20$ & $21-22$ & $23-24$ \\
\hline
\end{tabular}

Table 7. Offer $5 \%$ to all future customers when $P_{1}=20.000, P_{2}=50.000, \beta=0.999, m=$ $45, p=10$.

\begin{tabular}{cccccc}
\hline $\boldsymbol{r}_{1}$ & $\boldsymbol{n}_{1}$ & $\boldsymbol{r}_{2}$ & $\boldsymbol{n}_{2}$ & Profit $(\boldsymbol{E})$ & Offer \\
\hline 1 & 3 & 1 & 5 & 4.5480 & $5 \%$ \\
2 & 3 & 1 & 5 & 10.9007 & $5 \%$ \\
3 & 3 & 1 & 5 & 19.9805 & $5 \%$ \\
\hline
\end{tabular}

Table 8. Offer $10 \%$ to all future customers when $P_{1}=20.000, P_{2}=50.000, \beta=0.999, m=$ $45, p=10$.

\begin{tabular}{cccccc}
\hline$r_{1}$ & $n_{1}$ & $r_{2}$ & $n_{2}$ & Profit (£) & Offer \\
\hline 1 & 6 & 4 & 10 & 1.9790 & $10 \%$ \\
2 & 6 & 4 & 10 & 4.3508 & $10 \%$ \\
3 & 6 & 4 & 10 & 7.2222 & $10 \%$ \\
4 & 6 & 4 & 10 & 10.7365 & $10 \%$ \\
6 & 6 & 4 & 10 & 15.0873 & $10 \%$ \\
\hline
\end{tabular}

\subsection{Three Variants Case}

If there are 3 variant of products (5\%,10\% and $15 \%$ interest rates), then variant

5\% will be chosen if $\frac{r_{1}}{n_{1}} P_{1}>\frac{r_{1}}{n_{1}} \frac{r_{2}}{n_{2}} P_{2}$ and $\frac{r_{1}}{n_{1}} P_{1}>\frac{r_{1}}{n_{1}} \frac{r_{2}}{n_{2}} \frac{r_{3}}{n_{3}} P_{3}$. Variant $10 \%$ will 
be chosen if $\frac{r_{1}}{n_{1}} \frac{r_{2}}{n_{2}} P_{2}>\frac{r_{1}}{n_{1}} P_{1}$ and $\frac{r_{1}}{n_{1}} \frac{r_{2}}{n_{2}} P_{2}>\frac{r_{1}}{n_{1}} \frac{r_{2}}{n_{2}} \frac{r_{3}}{n_{3}} P_{3}$. Otherwise, variant $15 \%$ will be chosen if $\frac{r_{1}}{n_{1}} \frac{r_{2}}{n_{2}} \frac{r_{3}}{n_{3}} P_{3}>\frac{r_{1}}{n_{1}} P_{1}$ and $\frac{r_{1}}{n_{1}} \frac{r_{2}}{n_{2}} \frac{r_{3}}{n_{3}} P_{3}>\frac{r_{1}}{n_{1}} \frac{r_{2}}{n_{2}} P_{2}$.

Table 9 and Table 10 presented some of the results generated by the model for three variant of products. The bold in the row is the point when the switch of offers occurs. We choose $r_{1}=3$ and $n_{1}=16$ to represent a case where one's belief of the acceptance of variant $5 \%$ and variant $10 \%$ are $p_{1}=p_{2}=\frac{3}{8}$. The changes of offer shown are from variant $5 \%$ to variant $15 \%$.

Table 11 presents some of the results generated by the model for three variants of the product. The bold row is the point when the switch of offers occurs. We choose $r_{1}=1, n_{1}=1$ and $r_{3}=3, n_{3}=6$ to represent a case where one's belief of the acceptance of variant $5 \%$ and $15 \%$ are $p_{1}=1$ and $p_{3}=\frac{1}{2}$ respectively. The changes of offer are from variant $5 \%$ to variant $10 \%$.

Table 9. Part of results generated by the acceptance model when $P_{1}=10.000, P_{2}=25.000$, $P_{3}=35.000, \beta=0.5, m=6, p=6$.

\begin{tabular}{cccccccc}
\hline $\boldsymbol{r}_{1}$ & $\boldsymbol{n}_{1}$ & $\boldsymbol{r}_{2}$ & $\boldsymbol{n}_{2}$ & $\boldsymbol{r}_{3}$ & $\boldsymbol{n}_{3}$ & Profit $(\mathfrak{E})$ & Offer \\
\hline 6 & 16 & 6 & 16 & $0-3$ & 5 & 4.7471 & $5 \%$ \\
6 & 16 & 6 & 16 & 4 & 5 & 4.9972 & $15 \%$ \\
6 & 16 & 6 & 16 & 5 & 5 & 6.2305 & $15 \%$ \\
\hline
\end{tabular}

Table 10. Part of results generated by the acceptance model when $P_{1}=10.000, P_{2}=$ $25.000, P_{3}=35.000, \beta=0.5, m=6, p=6$.

\begin{tabular}{cccccccc}
\hline $\boldsymbol{r}_{1}$ & $\boldsymbol{n}_{1}$ & $\boldsymbol{r}_{2}$ & $\boldsymbol{n}_{2}$ & $\boldsymbol{r}_{3}$ & $\boldsymbol{n}_{3}$ & Profit (£) & Offer \\
\hline 6 & 16 & 6 & 16 & $0-12$ & 16 & 4.7471 & $5 \%$ \\
6 & 16 & 6 & 16 & 13 & 16 & 5.0671 & $15 \%$ \\
6 & 16 & 6 & 16 & 14 & 16 & 5.4522 & $15 \%$ \\
6 & 16 & 6 & 16 & 15 & 16 & 5.8412 & $15 \%$ \\
6 & 16 & 6 & 16 & 16 & 16 & 6.2305 & $15 \%$ \\
\hline
\end{tabular}

Table 11. Part of results generated by the acceptance model when $P_{1}=10.000, P_{2}=$ $25.000, P_{3}=35.000, \beta=0.5, m=6, p=6$.

\begin{tabular}{cccccccc}
\hline$r_{1}$ & $n_{1}$ & $r_{2}$ & $n_{2}$ & $r_{3}$ & $n_{3}$ & Profit $(\mathfrak{E})$ & Offer \\
\hline 1 & 1 & 1 & 5 & 3 & 6 & 19.6875 & $5 \%$ \\
1 & 1 & 2 & 5 & 3 & 6 & 20.6719 & $10 \%$ \\
1 & 1 & 3 & 5 & 3 & 6 & 29.5469 & $10 \%$ \\
1 & 1 & 4 & 5 & 3 & 6 & 39.3750 & $10 \%$ \\
1 & 1 & 5 & 5 & 3 & 6 & 49.2188 & $10 \%$ \\
\hline
\end{tabular}


Table 12 presents some of the results generated by the model for three variants of the product. The bold row is the point when the switch of offers occurs. We choose $r_{1}=4, n_{1}=11$ and $r_{2}=3, n_{2}=7$ to represent a case where one's belief of the acceptance of variant $5 \%$ and $10 \%$ are $p_{1}=\frac{4}{11}$ and $p_{2}=\frac{3}{7}$ respectively.

Table 13 and Table 14 present a case where one's belief of the acceptance of variant $5 \%$ with the ratio of $p_{1}=p_{2}=\frac{4}{5}$ and acceptance of variant $10 \%$ with the ratio of $p_{1}=p_{2}=\frac{3}{8}$ respectively for some of the belief points at which the offer decision changes.

Tables 15-17, presented here show that there is no any point of the switch of offers occurs. That is $r^{*}(n)$ does not exists in any state of $\left(r_{1}, n_{1}, \cdots, r_{m}, n_{m}\right)$. In Table 15, we choose $r_{2}=5, n_{2}=13$ and $r_{3}=4, n_{3}=14$ to represent a case where one's belief of the acceptance of variant $10 \%$ is $p_{2}=\frac{5}{13}$ and acceptance of variant $15 \%$ is $p_{3}=\frac{2}{7}$. In Table 16 , we choose $r_{2}=6, n_{2}=15$ and $r_{3}=3, n_{3}=12$ to represent a case where one's belief of the acceptance of variant $10 \%$ is $p_{2}=\frac{2}{5}$ and acceptance of variant $15 \%$ is $p_{3}=\frac{1}{4}$. In Table 17 , we choose $r_{2}=6, n_{2}=11$ and $r_{3}=3, n_{3}=4$ to represent a case where one's belief

Table 12. Part of results generated by the acceptance model when $P_{1}=10.000, P_{2}=$ 25.000, $P_{3}=35.000, \beta=0.5, m=6, p=6$.

\begin{tabular}{cccccccc}
\hline $\boldsymbol{r}_{1}$ & $\boldsymbol{n}_{1}$ & $\boldsymbol{r}_{2}$ & $\boldsymbol{n}_{2}$ & $\boldsymbol{r}_{3}$ & $\boldsymbol{n}_{3}$ & Profit (E) & Offer \\
\hline 4 & 11 & 3 & 7 & $0-9$ & 13 & 4.9934 & $10 \%$ \\
4 & 11 & 3 & 7 & 10 & 13 & 5.3483 & $15 \%$ \\
4 & 11 & 3 & 7 & 11 & 13 & 5.8748 & $15 \%$ \\
4 & 11 & 3 & 7 & 12 & 13 & 6.4076 & $15 \%$ \\
4 & 11 & 3 & 7 & 13 & 13 & 6.9412 & $10 \%$ \\
\hline
\end{tabular}

Table 13. Changing of offers when $r_{5}=4, n_{5}=5, \mathrm{r}_{10}=4, n_{10}=5, P_{1}=10.000, P_{2}=25.000$, $P_{3}=35.000, \beta=0.5, m=6, p=6$.

\begin{tabular}{ccccccccccccc}
\hline $\boldsymbol{r}_{15}$ & 0 & 1 & 2 & 3 & 4 & 5 & 6 & 7 & 8 & 9 & 10 & 11 \\
\hline $\boldsymbol{n}_{15}$ & 1 & 2 & $3-4$ & 5 & $6-7$ & 8 & 9 & $10-11$ & 12 & $13-14$ & 15 & 16
\end{tabular}

Table 14. Changing of offers when $r_{5}=6, n_{5}=16, \mathrm{r}_{10}=6, n_{10}=16, P_{1}=10.000, P_{2}=$ 25.000, $P_{3}=35.000, \beta=0.5, m=6, p=6$.

\begin{tabular}{cccccccccccccc}
\hline $\boldsymbol{r}_{15}$ & 0 & 1 & 2 & 3 & 4 & 5 & 6 & 7 & 8 & 9 & 10 & 11 & 12 \\
\hline $\boldsymbol{n}_{15}$ & 1 & 2 & 3 & $4-5$ & 6 & 7 & $8-9$ & 10 & 11 & $12-13$ & 14 & 15 & 16 \\
\hline
\end{tabular}


Table 15. Offer 5\% to all future customers when $P_{1}=10.000, P_{2}=25.000, P_{3}=35.000, \beta=$ $0.5, m=6, p=6$.

\begin{tabular}{cccccccc}
\hline $\boldsymbol{r}_{1}$ & $\boldsymbol{n}_{1}$ & $\boldsymbol{r}_{2}$ & $\boldsymbol{n}_{2}$ & $\boldsymbol{r}_{3}$ & $\boldsymbol{n}_{3}$ & Profit $(\mathfrak{E})$ & Offer \\
\hline 1 & 6 & 5 & 13 & 4 & 14 & 1.9625 & $5 \%$ \\
2 & 6 & 5 & 13 & 4 & 14 & 4.2954 & $5 \%$ \\
3 & 6 & 5 & 13 & 4 & 14 & 7.0948 & $5 \%$ \\
4 & 6 & 5 & 13 & 4 & 14 & 10.4834 & $5 \%$ \\
5 & 6 & 5 & 13 & 4 & 14 & 14.6163 & $5 \%$ \\
6 & 6 & 5 & 13 & 4 & 14 & 19.6875 & $5 \%$ \\
\hline
\end{tabular}

Table 16. Offer $10 \%$ to all future customers when $P_{1}=10.000, P_{2}=25.000, P_{3}=35.000, \beta=$ $0.5, m=6, p=6$.

\begin{tabular}{|c|c|c|c|c|c|c|c|}
\hline$r_{1}$ & $n_{1}$ & $r_{2}$ & $n_{2}$ & $r_{3}$ & $n_{3}$ & Profit $(\mathfrak{E})$ & Offer \\
\hline 1 & 10 & 6 & 15 & 3 & 12 & 1.1083 & $10 \%$ \\
\hline 2 & 10 & 6 & 15 & 3 & 12 & 2.3416 & $10 \%$ \\
\hline 3 & 10 & 6 & 15 & 3 & 12 & 3.7198 & $10 \%$ \\
\hline 4 & 10 & 6 & 15 & 3 & 12 & 5.2663 & $10 \%$ \\
\hline 5 & 10 & 6 & 15 & 3 & 12 & 7.0094 & $10 \%$ \\
\hline 6 & 10 & 6 & 15 & 3 & 12 & 8.9822 & $10 \%$ \\
\hline 7 & 10 & 6 & 15 & 3 & 12 & 11.2237 & $10 \%$ \\
\hline 8 & 10 & 6 & 15 & 3 & 12 & 13.7799 & $10 \%$ \\
\hline 9 & 10 & 6 & 15 & 3 & 12 & 16.7042 & $10 \%$ \\
\hline 10 & 10 & 6 & 15 & 3 & 12 & 20.0588 & $10 \%$ \\
\hline
\end{tabular}

Table 17. Offer $15 \%$ to all future customers when $P_{1}=10.000, P_{2}=25.000, P_{3}=35.000, \beta=$ $0.5, m=6, p=6$.

\begin{tabular}{cccccccc}
\hline $\boldsymbol{r}_{1}$ & $\boldsymbol{n}_{1}$ & $\boldsymbol{r}_{2}$ & $\boldsymbol{n}_{2}$ & $\boldsymbol{r}_{3}$ & $\boldsymbol{n}_{3}$ & Profit $(\mathfrak{E})$ & Offer \\
\hline 1 & 5 & 6 & 11 & 3 & 4 & 3.4935 & $15 \%$ \\
2 & 5 & 6 & 11 & 3 & 4 & 7.8071 & $15 \%$ \\
3 & 5 & 6 & 11 & 3 & 4 & 13.1930 & $15 \%$ \\
4 & 5 & 6 & 11 & 3 & 4 & 19.9823 & $15 \%$ \\
5 & 5 & 6 & 11 & 3 & 4 & 28.6029 & $15 \%$ \\
\hline
\end{tabular}

of the acceptance of variant $10 \%$ is $p_{2}=\frac{6}{11}$ and acceptance of variant $15 \%$ is $p_{3}=\frac{3}{4}$.

Table 18 shows that as $r_{1}$ increases but the rate $\frac{r_{1}}{n_{1}}$ and $\frac{r_{2}}{n_{2}}$ are fixed, the crucial value where one changes offers, $r_{1}^{*}\left(r_{2}, n_{2}\right)$, is monotonically non increasing. We also give results for the effect of more information (increment of 
Table 18. Effect of more information on the switch of offers.

\begin{tabular}{ccccccccccccccccccccc}
\hline$r_{1}, n_{1}$ & $r_{2,} n_{2}$ & $n_{3}$ & 1 & 2 & 3 & 4 & 5 & 6 & 7 & 8 & 9 & 10 & 11 & 12 & 13 & 14 & 15 & 16 & Changes of Offer \\
\hline 16,16 & 1,16 & & - & - & - & - & - & - & - & - & - & - & - & - & - & - & - & - & Remain $5 \%$ \\
16,16 & 4,16 & & 0 & 1 & 2 & 3 & 4 & 5 & 6 & 7 & 8 & 9 & 10 & 10 & 11 & 12 & 13 & 14 & $5 \%$ to $15 \%$ \\
16,16 & 8,16 & & $r_{2}^{*}\left(r_{3}, n_{3}\right)$ & 0 & 1 & 1 & 2 & 2 & 3 & 4 & 4 & 5 & 5 & 6 & 7 & 7 & 8 & 9 & 9 & $10 \%$ to $15 \%$ \\
16,16 & 14,16 & & 0 & 0 & 1 & 2 & 2 & 3 & 4 & 4 & 5 & 5 & 6 & 7 & 7 & 8 & 9 & 9 & $10 \%$ to $15 \%$ \\
16,16 & 15,16 & & 0 & 0 & 1 & 2 & 2 & 3 & 4 & 4 & 5 & 5 & 6 & 7 & 7 & 8 & 8 & 9 & $10 \%$ to $15 \%$ \\
16,16 & 16,16 & & 0 & 0 & 1 & 2 & 2 & 3 & 4 & 4 & 5 & 5 & 6 & 7 & 7 & 8 & 8 & 9 & $10 \%$ to $15 \%$ \\
\hline
\end{tabular}

$\left.r_{2}\right)$ in the table. The $r_{1}^{*}\left(r_{2}, n_{2}\right)$ for $n_{2}$ varying from 1 to 16 . Note that the hyphen $(-)$ in the table means that there is no changes of the offer occurs where Offer $5 \%$ is the only offer.

\subsection{Four Variants Case}

If there are 4 variants of products, then variant $5 \%$ will be chosen if $\frac{r_{1}}{n_{1}} P_{1}>\frac{r_{1}}{n_{1}} \frac{r_{2}}{n_{2}} P_{2}, \frac{r_{1}}{n_{1}} P_{1}>\frac{r_{1}}{n_{1}} \frac{r_{2}}{n_{2}} \frac{r_{3}}{n_{3}} P_{3}$, and $\frac{r_{1}}{n_{1}} P_{1}>\frac{r_{1}}{n_{1}} \frac{r_{2}}{n_{2}} \frac{r_{3}}{n_{3}} \frac{r_{4}}{n_{4}} P_{4}$. Variant $10 \%$ will be chosen if $\frac{r_{1}}{n_{1}} \frac{r_{2}}{n_{2}} P_{2}>\frac{r_{1}}{n_{1}} P_{1}, \frac{r_{1}}{n_{1}} \frac{r_{2}}{n_{2}} P_{2}>\frac{r_{1}}{n_{1}} \frac{r_{2}}{n_{2}} \frac{r_{3}}{n_{3}} P_{3}$, and

$\frac{r_{1}}{n_{1}} \frac{r_{2}}{n_{2}} P_{2}>\frac{r_{1}}{n_{1}} \frac{r_{2}}{n_{2}} \frac{r_{3}}{n_{3}} \frac{r_{4}}{n_{4}} P_{4}$. Variant $15 \%$ will be chosen if $\frac{r_{1}}{n_{1}} \frac{r_{2}}{n_{2}} \frac{r_{3}}{n_{3}} P_{3}>\frac{r_{1}}{n_{1}} P_{1}$,

$\frac{r_{1}}{n_{1}} \frac{r_{2}}{n_{2}} \frac{r_{3}}{n_{3}} P_{3}>\frac{r_{1}}{n_{1}} \frac{r_{2}}{n_{2}} P_{2}$, and $\frac{r_{1}}{n_{1}} \frac{r_{2}}{n_{2}} \frac{r_{3}}{n_{3}} P_{3}>\frac{r_{1}}{n_{1}} \frac{r_{2}}{n_{2}} \frac{r_{3}}{n_{3}} \frac{r_{4}}{n_{4}} P_{4}$. Otherwise, variant $20 \%$ will be chosen if $\frac{r_{1}}{n_{1}} \frac{r_{2}}{n_{2}} \frac{r_{3}}{n_{3}} \frac{r_{4}}{n_{4}} P_{4}>\frac{r_{1}}{n_{1}} P_{1}, \frac{r_{1}}{n_{1}} \frac{r_{2}}{n_{2}} \frac{r_{3}}{n_{3}} \frac{r_{4}}{n_{4}} P_{4}>\frac{r_{1}}{n_{1}} \frac{r_{2}}{n_{2}} P_{2}$, and $\frac{r_{1}}{n_{1}} \frac{r_{2}}{n_{2}} \frac{r_{3}}{n_{3}} \frac{r_{4}}{n_{4}} P_{4}>\frac{r_{1}}{n_{1}} \frac{r_{2}}{n_{2}} \frac{r_{3}}{n_{3}} P_{3}$.

Table 19 presents some of the results generated by the model for four variants of the product. The bold row is the point when the switch of offers occurs. We choose $r_{1}=2, n_{1}=2, r_{3}=1, n_{3}=7$, and $r_{4}=1, n_{4}=7$ to represent a case where one's belief of the acceptance of variant $5 \%, 15 \%$ and $20 \%$ are $p_{1}=1$ and $p_{3}=p_{4}=0$ respectively. The changes of offer are from variant $5 \%$ to variant $10 \%$.

Table 20 presents some of the results generated by the model for four variants of the products. The bold row is the point when the switch of offers occurs. We choose $r_{1}=4, n_{1}=6, r_{2}=2, n_{2}=6$ and $r_{4}=2, n_{4}=3$ to represent a case where one's belief of the acceptance of variant $5 \%, 10 \%$ and $20 \%$ are $p_{1}=p_{4}=\frac{2}{3}$ and $p_{2}=\frac{1}{3}$ respectively. The changes of offer shown is from variant $5 \%$ to variant $15 \%$.

Table 21 presents some of the results generated by the model for four variants of the products. The bold row is the point when the switch of offers occurs. We 
Table 19. Part of results generated by the acceptance model when $P_{1}=20.000, P_{2}=$ 50.000, $P_{3}=80.000, P_{4}=100.000, \beta=0.999, m=3, p=2$.

\begin{tabular}{cccccccccc}
\hline $\boldsymbol{r}_{1}$ & $\boldsymbol{n}_{1}$ & $\boldsymbol{r}_{2}$ & $\boldsymbol{n}_{2}$ & $\boldsymbol{r}_{3}$ & $\boldsymbol{n}_{3}$ & $\boldsymbol{r}_{4}$ & $\boldsymbol{n}_{4}$ & Profit (£) & Offer \\
\hline 2 & 2 & $1-2$ & 6 & 1 & 7 & 1 & 7 & 39.9800 & $5 \%$ \\
2 & 2 & 3 & 6 & 1 & 7 & 1 & 7 & 49.9750 & $10 \%$ \\
2 & 2 & 4 & 6 & 1 & 7 & 1 & 7 & 66.6333 & $10 \%$ \\
2 & 2 & 5 & 6 & 1 & 7 & 1 & 7 & 83.2917 & $10 \%$ \\
2 & 2 & 6 & 6 & 1 & 7 & 1 & 7 & 99.9500 & $10 \%$ \\
\hline
\end{tabular}

Table 20. Part of results generated by the acceptance model when $P_{1}=20.000, P_{2}=$ 50.000, $P_{3}=80.000, P_{4}=100.000, \beta=0.999, m=3, p=2$.

\begin{tabular}{cccccccccc}
\hline $\boldsymbol{r}_{1}$ & $\boldsymbol{n}_{1}$ & $\boldsymbol{r}_{2}$ & $\boldsymbol{n}_{2}$ & $\boldsymbol{r}_{3}$ & $\boldsymbol{n}_{3}$ & $\boldsymbol{r}_{4}$ & $\boldsymbol{n}_{4}$ & Profit $(\mathfrak{E})$ & Offer \\
\hline 4 & 6 & 2 & 6 & $1-2$ & 3 & 2 & 3 & 22.8576 & $5 \%$ \\
4 & $\mathbf{6}$ & $\mathbf{2}$ & $\mathbf{6}$ & $\mathbf{3}$ & $\mathbf{3}$ & $\mathbf{2}$ & $\mathbf{3}$ & $\mathbf{3 0 . 4 6 3 5}$ & $\mathbf{1 5 \%}$ \\
\hline
\end{tabular}

Table 21. Part of results generated by the acceptance model when $P_{1}=20.000, P_{2}=$ 50.000, $P_{3}=80.000, P_{4}=100.000, \beta=0.999, m=3, p=2$.

\begin{tabular}{cccccccccc}
\hline $\boldsymbol{r}_{1}$ & $\boldsymbol{n}_{1}$ & $\boldsymbol{r}_{2}$ & $\boldsymbol{n}_{2}$ & $\boldsymbol{r}_{3}$ & $\boldsymbol{n}_{3}$ & $\boldsymbol{r}_{4}$ & $\boldsymbol{n}_{4}$ & Profit (E) & Offer \\
\hline 5 & 6 & 1 & 5 & 6 & 7 & 6 & 7 & 30.9381 & $5 \%$ \\
$\mathbf{5}$ & $\mathbf{6}$ & 2 & $\mathbf{5}$ & $\mathbf{6}$ & $\mathbf{7}$ & $\mathbf{6}$ & $\mathbf{7}$ & $\mathbf{4 5 . 5 3 6 5}$ & $\mathbf{2 0 \%}$ \\
5 & 6 & 3 & 5 & 6 & 7 & 6 & 7 & 68.3430 & $20 \%$ \\
5 & 6 & 4 & 5 & 6 & 7 & 6 & 7 & 91.1750 & $20 \%$ \\
5 & 6 & 5 & 5 & 6 & 7 & 6 & 7 & 114.0324 & $20 \%$ \\
\hline
\end{tabular}

choose $r_{1}=5, n_{1}=6, r_{3}=6, n_{3}=7$, and $r_{4}=6, n_{4}=7$ to represent a case where one's belief of the acceptance of variant $5 \%, 10 \%$ and $20 \%$ are $p_{1}=\frac{5}{6}$ and $p_{3}=p_{4}=\frac{6}{7}$ respectively. The changes of offer shown is from variant $5 \%$ to variant $20 \%$.

Tables 22-24 shown that the changes of offer is from variant $10 \%$ to variant $15 \%$, variant $10 \%$ to variant $20 \%$ and variant $15 \%$ to variant $20 \%$ respectively.

Tables 25-28 presented here show no point for the switch of offers. That is $r_{t}^{*}\left(r_{t+1}, n_{t+1}, \cdots, r_{m}, n_{m}\right)$ does not exist in any state of $\left(r_{1}, n_{1}, \cdots, r_{m}, n_{m}\right)$.

Table 22. Part of results generated by the acceptance model when. $P_{1}=20.000, P_{2}=$ 50.000, $P_{3}=80.000, P_{4}=100.000, \beta=0.999, m=3, p=2$.

\begin{tabular}{cccccccccc}
\hline $\boldsymbol{r}_{1}$ & $\boldsymbol{n}_{1}$ & $\boldsymbol{r}_{2}$ & $\boldsymbol{n}_{2}$ & $\boldsymbol{r}_{3}$ & $\boldsymbol{n}_{3}$ & $\boldsymbol{r}_{4}$ & $\boldsymbol{n}_{4}$ & Profit (E) & Offer \\
\hline 6 & 6 & 3 & 5 & $1-3$ & 6 & 2 & 5 & 59.9700 & $10 \%$ \\
6 & 6 & 3 & 5 & 4 & 6 & 2 & 5 & 64.5389 & $15 \%$ \\
6 & 6 & 3 & 5 & 5 & 6 & 2 & 5 & 79.9600 & $15 \%$ \\
6 & 6 & 3 & 5 & 6 & 6 & 2 & 5 & 95.9520 & $15 \%$ \\
\hline
\end{tabular}


Table 23. Part of results generated by the acceptance model when $P_{1}=20.000, P_{2}=$ 50.000, $P_{3}=80.000, P_{4}=100.000, \beta=0.999, m=3, p=2$.

\begin{tabular}{cccccccccc}
\hline $\boldsymbol{r}_{1}$ & $\boldsymbol{n}_{1}$ & $\boldsymbol{r}_{2}$ & $\boldsymbol{n}_{2}$ & $\boldsymbol{r}_{3}$ & $\boldsymbol{n}_{3}$ & $\boldsymbol{r}_{4}$ & $\boldsymbol{n}_{4}$ & Profit (£) & Offer \\
\hline 3 & 4 & 3 & 7 & $1-3$ & 7 & 7 & 7 & 29.3439 & $10 \%$ \\
3 & 4 & $\mathbf{3}$ & $\mathbf{7}$ & 4 & 7 & 7 & 7 & 33.0465 & $20 \%$ \\
3 & 4 & 3 & 7 & 5 & 7 & 7 & 7 & 41.3082 & $20 \%$ \\
3 & 4 & 3 & 7 & 6 & 7 & 7 & 7 & 49.5698 & $20 \%$ \\
3 & 4 & 3 & 7 & 7 & 7 & 7 & 7 & 57.8314 & $20 \%$ \\
\hline
\end{tabular}

Table 24. Part of results generated by the acceptance model when $P_{1}=20.000, P_{2}=$ 50.000, $P_{3}=80.000, P_{4}=100.000, \beta=0.999, m=3, p=2$.

\begin{tabular}{cccccccccc}
\hline $\boldsymbol{r}_{1}$ & $\boldsymbol{n}_{1}$ & $\boldsymbol{r}_{2}$ & $\boldsymbol{n}_{2}$ & $\boldsymbol{r}_{3}$ & $\boldsymbol{n}_{3}$ & $\boldsymbol{r}_{4}$ & $\boldsymbol{n}_{4}$ & Profit (E) & Offer \\
\hline 4 & 6 & 4 & 6 & 4 & 6 & $0-4$ & 6 & 40.9416 & $15 \%$ \\
4 & 6 & 4 & 6 & 4 & 6 & 5 & 6 & 42.6443 & $20 \%$ \\
4 & 6 & 4 & 6 & 4 & 6 & 6 & 6 & 50.7725 & $20 \%$ \\
\hline
\end{tabular}

Table 25. Offer 5\% to all future customers when $P_{1}=20.000, P_{2}=50.000, P_{3}=80.000, P_{4}=$ $100.000, \beta=0.999, m=3, p=2$.

\begin{tabular}{cccccccccc}
\hline $\boldsymbol{r}_{\mathbf{1}}$ & $\boldsymbol{n}_{\mathbf{1}}$ & $\boldsymbol{r}_{2}$ & $\boldsymbol{n}_{\mathbf{2}}$ & $\boldsymbol{r}_{\mathbf{3}}$ & $\boldsymbol{n}_{\mathbf{3}}$ & $\boldsymbol{r}_{4}$ & $\boldsymbol{n}_{4}$ & Profit (E) & Offer \\
\hline 1 & 3 & 1 & 4 & 5 & 7 & 6 & 8 & 9.9967 & $5 \%$ \\
2 & 3 & 1 & 4 & 5 & 7 & 6 & 8 & 23.3233 & $5 \%$ \\
3 & 3 & 1 & 4 & 5 & 7 & 6 & 8 & 39.9800 & $5 \%$ \\
\hline
\end{tabular}

Table 26. Offer $10 \%$ to all future customers when $P_{1}=20.000, P_{2}=50.000, P_{3}=80.000, P_{4}=$ $100.000, \beta=0.999, m=3, p=2$.

\begin{tabular}{cccccccccc}
\hline $\boldsymbol{r}_{1}$ & $\boldsymbol{n}_{1}$ & $\boldsymbol{r}_{2}$ & $\boldsymbol{n}_{\mathbf{2}}$ & $\boldsymbol{r}_{3}$ & $\boldsymbol{n}_{3}$ & $\boldsymbol{r}_{4}$ & $\boldsymbol{n}_{4}$ & Profit (£) & Offer \\
\hline 1 & 3 & 5 & 6 & 4 & 7 & 2 & 7 & 20.8264 & $10 \%$ \\
2 & 3 & 5 & 6 & 4 & 7 & 2 & 7 & 48.5903 & $10 \%$ \\
3 & 3 & 5 & 6 & 4 & 7 & 2 & 7 & 83.2917 & $10 \%$ \\
\hline
\end{tabular}

Table 27. Offer $15 \%$ to all future customers when $P_{1}=20.000, P_{2}=50.000, P_{3}=80.000$, $P_{4}=100.000, \beta=0.999, m=3, p=2$.

\begin{tabular}{cccccccccc}
\hline $\boldsymbol{r}_{1}$ & $\boldsymbol{n}_{1}$ & $\boldsymbol{r}_{2}$ & $\boldsymbol{n}_{2}$ & $\boldsymbol{r}_{3}$ & $\boldsymbol{n}_{3}$ & $\boldsymbol{r}_{4}$ & $\boldsymbol{n}_{4}$ & Profit (£) & Offer \\
\hline 1 & 4 & 2 & 7 & 2 & 2 & 4 & 6 & 7.9977 & $15 \%$ \\
2 & 4 & 2 & 7 & 2 & 2 & 4 & 6 & 18.2789 & $15 \%$ \\
3 & 4 & 2 & 7 & 2 & 2 & 4 & 6 & 30.8434 & $15 \%$ \\
4 & 4 & 2 & 7 & 2 & 2 & 4 & 6 & 45.6914 & $15 \%$ \\
\hline
\end{tabular}


Table 28. Offer $20 \%$ to all future customers when $P_{1}=20.000, P_{2}=50.000, P_{3}=80.000, P_{4}=$ $100.000, \beta=0.999, m=3, p=2$.

\begin{tabular}{cccccccccc}
\hline $\boldsymbol{r}_{1}$ & $\boldsymbol{n}_{1}$ & $\boldsymbol{r}_{2}$ & $\boldsymbol{n}_{2}$ & $\boldsymbol{r}_{3}$ & $\boldsymbol{n}_{3}$ & $\boldsymbol{r}_{4}$ & $\boldsymbol{n}_{4}$ & Profit (£) & Offer \\
\hline 1 & 5 & 1 & 5 & 5 & 5 & 4 & 4 & 5.5096 & $20 \%$ \\
2 & 5 & 1 & 5 & 5 & 5 & 4 & 4 & 12.5288 & $20 \%$ \\
3 & 5 & 1 & 5 & 5 & 5 & 4 & 4 & 21.0576 & $20 \%$ \\
4 & 5 & 1 & 5 & 5 & 5 & 4 & 4 & 31.0960 & $20 \%$ \\
5 & 5 & 1 & 5 & 5 & 5 & 4 & 4 & 42.6440 & $20 \%$ \\
\hline
\end{tabular}

\section{Conclusion}

From the results, we can clearly see that there is at most one point of switch offers. No matter how many variants of the product offered, the switching offer will not more than one. Based on this observation, the model can tell the best offer to extend to the next customer in an efficient manner and maximise the profit earned. Hence the model is able to identify the best offer for variants of credit cards. Further research would be to test this on different financial products like mortgages.

\section{Acknowledgements}

This research is supported by the Research University Grant Scheme (RUGS) 05-02-12-1869RU.

\section{Disclosure Policy}

The authors declare that there is no conflict of interest regarding the publication of this paper.

\section{References}

[1] Stango, V. (2000) Competition and Pricing in the Credit Card Market. The Review of Economics and Statistics, 82, 499-508. https://doi.org/10.1162/003465300558858

[2] Grodzicki, D. (2012) The Evolution of Competition in the Credit Card Market. Working paper. Stanford University, Palo Alto, CA.

[3] Keeney, R.L. and Oliver, R.M. (2005) Designing Win-Win Financial Loan Products for Consumers and Business. Journal of the Operational Research Society, 56, 1030-1040. https://doi.org/10.1057/palgrave.jors.2601992

[4] Seow, H.-V. and Thomas, L.C. (2006) Using Adaptive Learning in Credit Scoring to Estimate Take-Up Probability Distribution. European Journal of Operational Research, 173, 880-892. https://doi.org/10.1016/j.ejor.2005.06.058

[5] Ma, P., Crook, J. and Ansell, J. (2010) Modelling Take-Up and Profitability. Journal of the Operational Research Society, 61, 430-442.

https://doi.org/10.1057/jors.2009.33

[6] Bellman, R. (1957) Dynamic Programming. Princeton University Press, Princeton, NJ.

[7] Puterman, M.L. (1994) Markov Decision Process: Discrete Stochastic Dynamic Programming. John Wiley \& Sons, Inc., Hoboken, NJ. 